International Mathematical Forum, 2, 2007, no. 64, 3191 - 3198

\title{
DEA Efficiency Analysis with Identifying Efficient and Full-Inefficient Frontier
}

\author{
G. R. Jahanshahloo ${ }^{a}$, M. Zohrehbandian ${ }^{b}$, F. Hosseinzadeh Lotfi ${ }^{a} 1$ \\ M. Ghadimi ${ }^{b}$, M. Ahadzadeh Namin ${ }^{a}$ and M. Hokkamzadeh ${ }^{b}$ \\ ${ }^{a}$ Department of Mathematics, Science \& Research Branch \\ Islamic Azad University, Tehran, Iran \\ ${ }^{b}$ Department of Mathematics, Islamic Azad University \\ P.O. Box 31485-313, Karaj, Iran
}

\begin{abstract}
Data envelopment analysis (DEA) is a mathematical programming technique for identifying relative efficiency scores of decision making units (DMUs). Recently, Amirteimoori (2007) Introduced an alternative efficiency measure based on efficient and anti-efficient frontiers. In this paper we introduce a new computational framework for identifying full-efficient and inefficient frontier of production possibility set (PPS) in DEA models with variable return to scale. This facets apply in finding full-efficient and inefficient DMUs, sensitivity and stability analysis, ranking, and etc.
\end{abstract}

Keywords: Data Envelopment Analysis; VRS, Efficient frontier, Fullinefficient frontier

\section{Introduction}

Data envelopment analysis(DEA), originally proposed by Charnes, et al. (1978), has became one of the most widely used methods in management sciences. DEA is a non parametric technique for measuring and evaluating the relative efficiency of DMUs which stand of decision making units with several inputs and outputs. Classical DEA models rely on the assumption that inputs have to be minimized and outputs have to be maximized.The performance of a DMU depends only on the identified efficient frontier characterized by the DMUs with an unity efficiency score. The efficiency of a DMU is a scaler

\footnotetext{
${ }^{1}$ Corresponding author. e-mail: hosseinzadeh_lotf@@yahoo.com
} 
measure ranging between zero and one. This scaler value is measured through a linear programming model (see[2,3]).

A DMU is efficient if and only if it is not possible to improve some of its input or outputs without worsening some of its other inputs or outputs (Parato-Koopmans Efficiency). The above Definition is then equivalent to asserting that a DMU is efficient if and only if it is not dominated by some other DMU (or combination of DMUs) with which it can be compared.

This orientation means that we are restricting attention to "technical" aspects of efficiency (sometimes referred to as "waste").

The plan for the rest of this paper is as follows. Section 2 formalized the data, notation, assumptions and formal expression of the concepts with which we deal. In section 3, we present an approach for identifying the equations of full-inefficient frontier and describe it by a simple example. In section 4 . Finally, section 5 draws the conclusive remarks.

\section{Terminology}

We assume that there are n DMUs to be evaluated indexed by $j=1,2, \ldots, n$ and each DMU is assumed to produce $\mathrm{s}$ different outputs from $\mathrm{m}$ different inputs, where the observed inputs and output vectors of $D M U_{j}$ are depicted as $X_{j}=\left(x_{1 j}, \ldots, x_{m j}\right)$ and $Y_{j}=\left(y_{1 j}, \ldots, y_{s j}\right)$ respectively. The production possibility set of obviously most widely used DEA model, BCC, is defined as vectors $(X, Y)$ as follows:

$$
T_{v}=\left\{(X, Y)^{T} \mid Y \leq \sum_{j=1}^{n} \lambda_{j} Y_{j}, X \geq \sum_{j=1}^{n} \lambda_{j} X_{j}, \sum_{j=1}^{n} \lambda_{j}=1, \lambda_{j} \geq 0, j=1, \ldots, n\right\}
$$

We define $A=\left\{\left(x_{j}, y_{j}\right) ; j=1, \ldots, n\right\}$ and $C(A) \subseteq R^{m+s}$ as the convex hull of all the DMUs and $F(S)$, full-inefficient frontier as follows.

$$
F(s)=\left\{(X, Y) \in T_{v} \mid \exists(\bar{X}, \bar{Y}) ;\left((-\bar{X}, \bar{Y}) \geq_{\neq}(-X, Y) \Rightarrow(\bar{X}, \bar{Y}) \notin C(A)\right)\right\}
$$

$\mathrm{F}(\mathrm{S})$ called full-inefficient frontier. Each DMU belonged to $\mathrm{F}(\mathrm{S})$ is called a full-inefficient DMU. In other words $D M U_{j}, \quad j \in\{1, \ldots, n\}$ is a full-inefficient $\mathrm{DMU}$ if there is no other point in $\mathrm{C}(\mathrm{A})$ which is dominated by $D M U_{j}$. 
The following model evaluates the radial distance of $D M U_{0}$ from $F(s)$

$$
\begin{array}{lll}
\text { Max } & \psi \\
\text { s.t. } & -\sum_{j=1}^{n} \lambda_{j} x_{i j} \leq-\psi x_{i 0}, & i=1, \ldots, m \\
& \sum_{j=1}^{n} \lambda_{j} y_{r j} \leq y_{r 0}, & \\
& \sum_{j=1}^{n} \lambda_{j}=1 & \\
& \lambda_{j} \geq 0, & j=1, \ldots, s
\end{array}
$$

Theorem 1. $\left(X_{0}, Y_{0}\right)$ is full-inefficient if and only if in model $(2), \psi^{*}=1$ and the optimal objective value of the following model is zero:

$$
\begin{array}{lll}
\operatorname{Max} & \sum_{i=1}^{m} s_{i}^{-}+\sum_{r=1}^{s} s_{r}^{+} & \\
\text {s.t. } & -\sum_{j=1}^{n} \lambda_{j} x_{i j}-s_{i}^{-}=-\psi^{*} x_{i 0}, & i=1, \ldots, m \\
& \sum_{j=1}^{n} \lambda_{j} y_{r j}+s_{r}^{+}=y_{r 0}, & \\
& \\
& \sum_{j=1}^{n} \lambda_{j}=1 & \\
& \\
\lambda_{j} \geq 0, & j=1, \ldots, s \\
& s_{i}^{-}, s_{r}^{+} \geq 0 & i=1, \ldots, m, r=1, \ldots, s
\end{array}
$$

where $s_{i}^{-}$and $s_{r}^{+}$are $\mathrm{m}$ and s slack vectors, respectively, and 1 is a vector of $1 \mathrm{~s}$.

proof. (see Jahanshahloo et al. 2006)

In the next section we will introduce the procedure for finding the full-inefficient frontier, that will reducible for finding the equation of full-efficient frontier facility.

\section{Identifying the equations of full-inefficient frontier}

We use an iterative procedure for finding the full-inefficient frontier. We start with one of the facets of $\mathrm{F}(\mathrm{S})$. For finding the equation of this facet, we solve the LP model (2) for an arbitrary DMU, e.g. $D M U_{0}$. Suppose that $D M U_{0}^{*}=\left(\psi^{*} x_{0}, y_{0}\right)$ is the projection point of $D M U_{0}$ on $\mathrm{F}(\mathrm{s})$. The next step is to obtain the equation of a facet of $\mathrm{F}(\mathrm{S})$ containing $D M U_{0}^{*}$. This can be 
done by using the dual of LP model (2), as follows.

$$
\begin{array}{ll}
\text { Min } & \sum_{r=1}^{s} u_{r} y_{r 0}+w \\
\text { s.t. } & \sum_{r=1}^{s} u_{r} y_{r j}-\sum_{i=1}^{m} v_{i} x_{i j}+w \leq 0 \quad j=1, \ldots, n \\
& \sum_{i=1}^{m} v_{i} x_{i 0}=1 \\
& u, v \geq 0
\end{array}
$$

$\left(-v^{*}, u^{*}, w^{*}\right)$ is the normal of the hyperplane of $\mathrm{F}(\mathrm{s})$ which contains the $D M U_{0}^{*}$ (GF in fig.1). However, to obtain optimal dual variables, instead of solving LP model (4) we can use data from the optimal simplex tableau obtained by solving LP model (2) which has been already solved. Negative values of optimal dual variables are in the reduced cost row under the slack variables in the optimal simplex tableau of LP model (2).

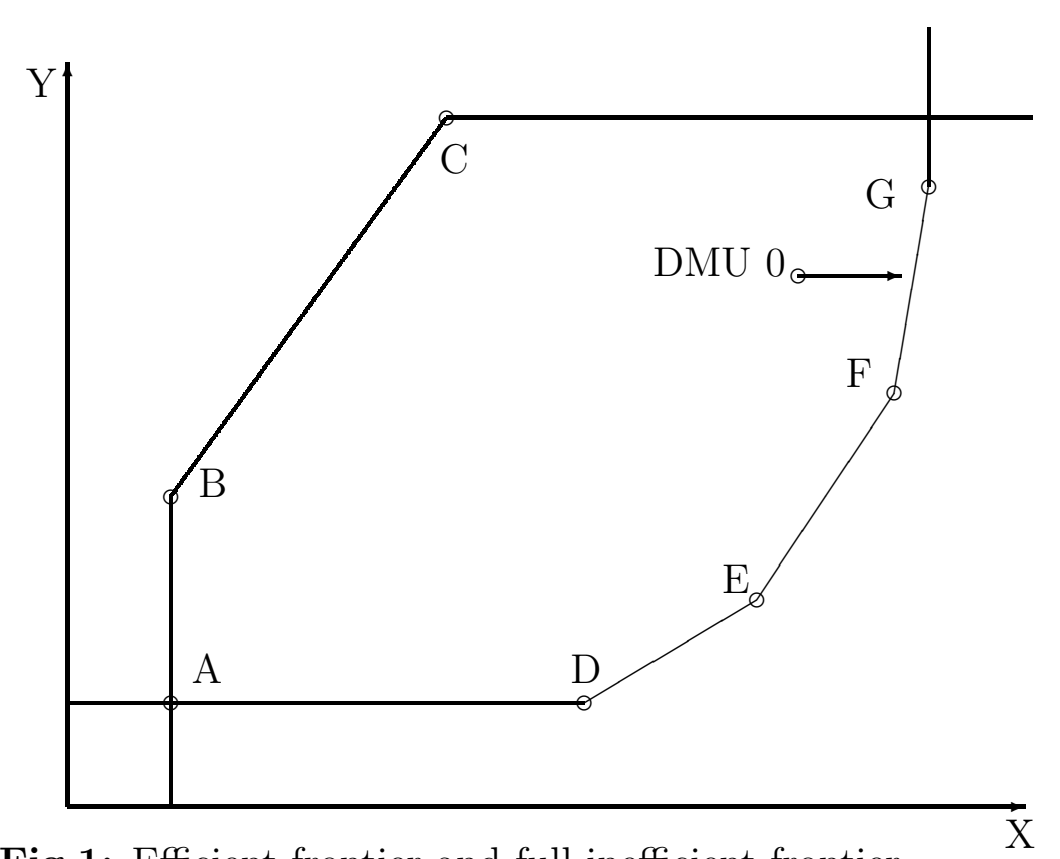

Fig.1: Efficient frontier and full-inefficient frontier

For finding other facets of F(s)(FE, ED in fig.1) we use an iterative procedure. In the iterative part of the procedure we also use data from the obtained simplex tableau. To move to adjacent facet we have to perform a dual pivot step in optimal simplex of LP model (2), i.e. one variable leaves and new one enters the basis. In selecting variable (one of $\lambda_{\mathrm{s}}$ or slack variables, not the $\psi$ ) which will leave the basis one condition must be satisfied.

There must be negative coefficients in the corresponding row in the optimal simplex tableau. 
Then ,by performing a dual pivot step, a new optimal basis (thus a new facet) is obtained and we repeat the procedure with a new facet. we select basis with an arborescent method, therefore at the end of the process we obtained all of the full-inefficient facets of PPS.

\section{A numerical example}

In order to motivate our approach we apply a simple example involving just eight DMUs, each using one input to produce one output; see table 1. The efficient and inefficient frontier defined on the raw data from table 1 , is depicted in figure 2 .

Table 1 Raw Data.

\begin{tabular}{lllllllll}
\hline & $D M U_{1}$ & $D M U_{2}$ & $D M U_{3}$ & $D M U_{4}$ & $D M U_{5}$ & $D M U_{6}$ & $D M U_{7}$ & $D M U_{8}$ \\
\hline X-input & 1.5 & 4 & 5.5 & 7.5 & 10 & 12 & 10 & 12.5 \\
Y-output & 4.5 & 5.5 & 10 & 1.5 & 3 & 6 & 8 & 9.5 \\
\hline
\end{tabular}

We start the procedure by solving LP model (2) for $D M U_{7}$. We have:

$$
\begin{array}{ll}
\operatorname{Max} & \psi \\
\text { s.t. } & -1.5 \lambda_{1}-4 \lambda_{2}-5.5 \lambda_{3}-7.5 \lambda_{4}-10 \lambda_{5}-12 \lambda_{6}-10 \lambda_{7}-12.5 \lambda_{8} \leq-10 \psi \\
& 4.5 \lambda_{1}+5.5 \lambda_{2}+10 \lambda_{3}+1.5 \lambda_{4}+3 \lambda_{5}+6 \lambda_{6}+8 \lambda_{7}+9.5 \lambda_{8} \leq 8 \\
& \lambda_{1}+\lambda_{2}+\lambda_{3}+\lambda_{4}+\lambda_{5}+\lambda_{6}+\lambda_{7}+\lambda_{8}=1 \\
& \beta, \lambda_{j} \geq 0, \quad j=1, \ldots, 8
\end{array}
$$

\begin{tabular}{|c|c|c|c|c|c|c|c|c|c|c|c|c|c|}
\hline$B$ & $\psi$ & $\lambda_{1}$ & $\lambda_{2}$ & $\lambda_{3}$ & $\lambda_{4}$ & $\lambda_{5}$ & $\lambda_{6}$ & $\lambda_{7}$ & $\lambda_{8}$ & $s_{1}$ & $s_{2}$ & $s_{a}$ & $R H S$ \\
\hline$c_{j}-z_{j}$ & 0 & 1.029 & 0.793 & 0.707 & 0.386 & 0.157 & 0 & 0.229 & 0 & 0.1 & 0.014 & $\mathrm{M}+1.114$ & 1.229 \\
\hline$\psi$ & 1 & 1.029 & 0.793 & 0.707 & 0.386 & 0.157 & 0 & 0.229 & 0 & 0.1 & 0.014 & 1.114 & 1.229 \\
\hline$\lambda_{8}$ & 0 & -0.429 & -0.143 & 1.143 & -1.286 & -0.857 & 0 & 0.571 & 1 & 0 & 0.286 & -1.714 & 0.571 \\
\hline$\lambda_{6}$ & 0 & 1.429 & 1.143 & -0.143 & 2.286 & 1.857 & 1 & 0.429 & 0 & 0 & -0.286 & 2.714 & 0.429 \\
\hline
\end{tabular}

The optimal value of LP model (5) is $\psi^{*}=1.229$ with variables $\psi, \lambda_{8}$ and $\lambda_{6}$ in the optimal basis, $\lambda_{8}^{*}=0.571$ and $\lambda_{6}^{*}=0.429$. The projection point is $D M U_{7}^{*}=(12.29,8)^{T}$, and the optimal simplex tableau is

Tableau 1 Optimal simplex tableau of Step 1 


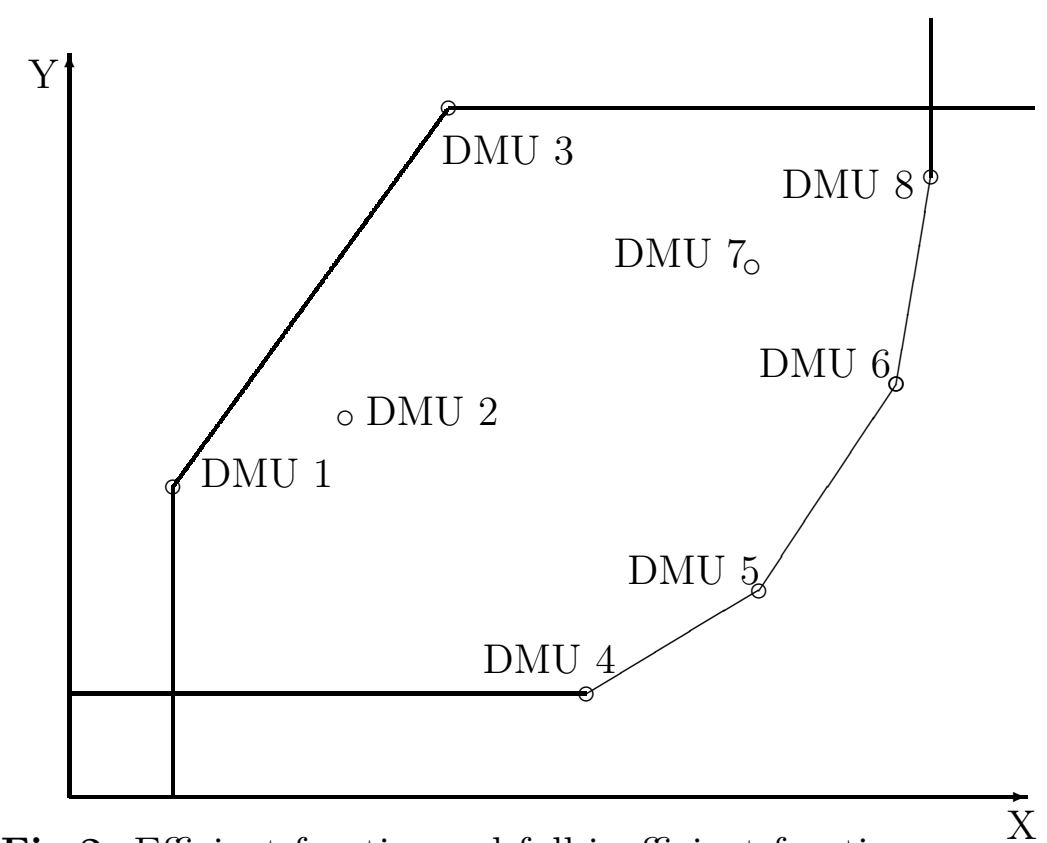

Fig.2: Efficient frontier and full-inefficient frontier

The optimal dual variables, i.e., $v^{*}=0.1, u^{*}=0.014, w^{*}=1.114$, are coefficients of the facet which contain the projection point $\left(D M U_{7}^{*}\right)$. The equation of this facet which contains the $D M U_{8}$ and $D M U_{6}$ is as follows:

$$
-0.1 x+0.014 y+1.114=0
$$

We should pivot to the adjacent facet. we have negative coefficients in the second and third row, therefore we deduce that two pivot step are possible. First, we can pivot such that $\lambda_{6}$ leaves the basis and $s_{2}$ enters the basis, thus obtaining new basis $B_{11}=\left\{\psi, \lambda_{8}, s_{2}\right\}$. Second, we can pivot such that $\lambda_{8}$ leaves and $\lambda_{5}$ enters the basis, thus obtaining basis $B_{12}=\left\{\psi, \lambda_{5}, \lambda_{6}\right\}$.

In the second step we perform first pivot step i.e., $\lambda_{6}$ leaves the basis and $s_{2}$ enters the basis and producing new simplex tableau as follows:

\begin{tabular}{llllllllllllll}
\hline$B$ & $\psi$ & $\lambda_{1}$ & $\lambda_{2}$ & $\lambda_{3}$ & $\lambda_{4}$ & $\lambda_{5}$ & $\lambda_{6}$ & $\lambda_{7}$ & $\lambda_{8}$ & $s_{1}$ & $s_{2}$ & $s_{a}$ & $R H S$ \\
\hline$c_{j}-z_{j}$ & 0 & 1.1 & 0.85 & 0.7 & 0.5 & 0.25 & 0.05 & 0.25 & 0 & 0.1 & 0 & $\mathrm{M}+1.25$ & 1.25 \\
$\psi$ & 1 & 1.1 & 0.85 & 0.7 & 0.5 & 0.25 & 0.05 & 0.25 & 0 & 0.1 & 0 & 1.25 & 1.25 \\
$\lambda_{8}$ & 0 & 1 & 1 & 1 & 1 & 1 & 1 & 1 & 1 & 0 & 0 & 1 & 1 \\
$s_{2}$ & 0 & -5 & -4 & 0.5 & -8 & -6.5 & -3.5 & -1.5 & 0 & 0 & 1 & -9.5 & -1.5 \\
\hline
\end{tabular}

Tableau 2 Optimal simplex tableau after pivoting in the first Step

From tableau 2 we obtain the new dual variables $v=0.1, u=0, w=1.250$. Then the equation of the new facet is

$$
-0.1 x+1.25=0
$$

Since in tableau 2 the only negative coefficient is on the third row and with dual simplex pivoting will only reverse the previous pivoting. This implies that 
we can not pivot to some new facet.

Hence, we proceed with basis $B_{12}=\left\{\psi, \lambda_{5}, \lambda_{6}\right\}$ i.e., $\lambda_{8}$ leaves and $\lambda_{5}$ enters the basis in tableau 1 , and producing new simplex tableau as follows:

\begin{tabular}{llllllllllllll}
\hline$B$ & $\psi$ & $\lambda_{1}$ & $\lambda_{2}$ & $\lambda_{3}$ & $\lambda_{4}$ & $\lambda_{5}$ & $\lambda_{6}$ & $\lambda_{7}$ & $\lambda_{8}$ & $s_{1}$ & $s_{2}$ & $s_{a}$ & $R H S$ \\
\hline$c_{j}-z_{j}$ & 0 & 0.95 & 0.767 & 0.917 & 0.150 & 0 & 0 & 0.333 & 0.183 & 0.1 & 0.067 & $\mathrm{M}+0.8$ & 1.333 \\
$\psi$ & 1 & 0.95 & 0.767 & 0.917 & 0.150 & 0 & 0 & 0.333 & 0.183 & 0.1 & 0.067 & 0.8 & 1.333 \\
$\lambda_{5}$ & 0 & 0.5 & 0.167 & -1.333 & 1.5 & 1 & 0 & -0.667 & -1.167 & 0 & -0.333 & 2 & -0.667 \\
$\lambda_{6}$ & 0 & 0.5 & 0.833 & 2.333 & -0.5 & 0 & 1 & 1.667 & 2.167 & 0 & 0.333 & -1 & 1.667 \\
\hline
\end{tabular}

Tableau 3 Simplex tableau after pivoting in the second Step

Dual variables are $v=0.1, u=0.067, w=0.8$. Then the equation of the new facet is

$$
-0.1 x+0.067 y+0.8=0
$$

we can pivot to the adjacent facet and producing new simplex tableau as follows:

\begin{tabular}{llllllllllllll}
\hline$B$ & $\psi$ & $\lambda_{1}$ & $\lambda_{2}$ & $\lambda_{3}$ & $\lambda_{4}$ & $\lambda_{5}$ & $\lambda_{6}$ & $\lambda_{7}$ & $\lambda_{8}$ & $s_{1}$ & $s_{2}$ & $s_{a}$ & $R H S$ \\
\hline$c_{j}-z_{j}$ & 0 & 1.1 & 1.017 & 1.617 & 0 & 0 & 0.3 & 0.833 & 0.833 & 0.1 & 0.167 & $\mathrm{M}+0.5$ & 1.833 \\
$\psi$ & 1 & 1.1 & 1.017 & 1.617 & 0 & 0 & 0.3 & 0.833 & 0.833 & 0.1 & 0.167 & 0.5 & 1.833 \\
$\lambda_{5}$ & 0 & 2 & 2.667 & 5.667 & 0 & 1 & 3 & 4.333 & 5.333 & 0 & 0.667 & -1 & 4.333 \\
$\lambda_{4}$ & 0 & -1 & -1.667 & -4.667 & 1 & 0 & -2 & -3.333 & -4.333 & 0 & -0.667 & 2 & -3.333 \\
\hline
\end{tabular}

Tableau 4 Optimal simplex tableau of Step 3

Dual variables are $v=0.1, u=0.167, w=0.5$ and the equation of the new facet is

$$
-0.1 x+0.167 y+0.5=0
$$

In tableau 4 the only negative coefficient is on the third row and with dual simplex pivoting will only reverse the previous pivoting. This implies that we can not pivot to some new facet. Hence, we obtain all facet of $\mathrm{F}(\mathrm{s})$.

\section{Conclusion}

We have proposed and approach to finding the equation of full- inefficient frontier in BCC model. at first we obtained the projection point of $D M U_{0}$ on full-inefficient frontier. We also use Primal-Dual relationship, to obtain the equation of the facet without solving the dual LP model.Each DMU belonged to Efficient facets is efficient DMU and Each DMU belonged to full-efficient facets is a full-inefficient DMU. But for DMUs that belonged to intersection efficient and full-inefficient facet(like(A) in fig.1), we can't make any decision about it.

We can use this method for CCR model and will reducible for finding the equation of efficient frontier whit solving BCC model instead of solving LP $\operatorname{model}(2)$. 


\section{References}

[1] Amirteimoori A (2007)"DEA efficiency analysis: Efficient and antiefficient frontier" Applied Mathematics and Computation,186, 10-16.

[2] Banker R.D., Cooper W.W., Rhodes E. (1984) "Some models for estimating technical and scale efficiencies in data envelopment analysis " European Journal of Management Science 30 (1984)14078-1092.

[3] Charnes A., Cooper W.W., Rhodes E. (1978) "Measuring the efficiency of decision making units" European Journal of Operational Research, 2, 429-444.

[4] Jahanshahloo G.R, Afzalinejad M (2006) "A ranking method based on a full-inefficient frontier" ,Applied Mathematical modelling 30, 248-260.

Received: September 5, 2007 